Research Article

\title{
Hematological profile in typhoid fever
}

\author{
Shrivastava $K^{1}$, Vahikar $S^{2}$, Mishra $\mathbf{V}^{3}$ \\ ${ }^{1}$ Dr Kanchan Shrivastava, Assistant Professor Department of Pathology, BRD Medical College, Formally Lecturer, \\ Pathology Department, GSVM Medical College, Kanpur. ${ }^{2}$ Dr Shilpa Vahikar, Associated Professor, Department of \\ Pathology, BRD Medical College, Gorakhpur, ${ }^{3}$ Dr Vandana Mishra, Associated Professor, Department of Pathology, \\ GSVM Medical College, Kanpur, UP, India
}

Address for correspondence: Dr Kanchan Shrivastava, Email: kanchanshrivastava11@gmail.com

\begin{abstract}
Introduction: Typhoid fever is an acute infection of the blood and intestinal system caused by the bacterium Salmonella typhi. In India S. typhi and S.paratyphi are the common agents of enteric fever. Complications of these lesions are hemorrhage and intestinal perforation. Carrier state is common and bacilli are found in blood, feces and urine. As there is massive multiplication of bacilli in blood stream, this paper aims to study the frequency and severity of hematological changes in patients of typhoid fever with special reference to thrombocytopenia. Methodology: This study was a hospital based descriptive study conducted on OPD and IPD patients of LLR Hospital Kanpur who presented to Emergency Pathology Lab, GSVM Medical College Kanpur. The study design was cross sectional. Study was designed to include demographics, clinical information and hematological changes observed in each patient of typhoid fever using widal test or positive culture. The data was analyzed to study the changes in hematological parameters in these patients. Result: The study revealed the sex ratio to be almost unity and maximum cases were seen in 11-20 year age group. Thrombocytopenia was observed in a significant $39.7 \%$ and leucopenia in $11.6 \%$ of cases. Mean PCV was reduced to $29.6 \%$ and mean neutropil percentage was reduced to $28 \%$ and mean lymphocyte percentage of was $59 \%$ showed relative lymphocytosis. Conclusion: Typhoid fever causes significant hematological changes which could be helpful in diagnosis. Thrombocytopenia is common in typhoid but this association is not well recognized. Awareness of this associate on could be useful in diagnosis of typoid fever more so in under resourced endemic regions in developing countries
\end{abstract}

Key words: Typhoid, Thrombocytopenia, Leucopenia

\section{Introduction}

Typhoid fever constitutes a major public health problem in many developing countries of the world and it has also been increasingly reported from the developed countries. Almost $80 \%$ of cases and death occur in Asia[1]. Typhoid fever is an acute infection of the blood and intestinal systems caused by the bacterium Salmonella typhi. Following ingestion, there is an incubation period of about $10-14$ days[2]. It is a severe multisystemic illness characterized by the classic prolonged fever, sustained bacteremia without endothelial or endocardial involvement. The hallmark of typhoid fever is the invasion of and multiplication within the mononuclear phagocytic cells in the liver, spleen, lymph nodes and payers patches of ileum. [3].

Manuscript received: $11^{\text {th }}$ Nov 2015

Reviewed: $24^{\text {th }}$ Nov 2015

Author Corrected: $14^{\text {th }}$ Dec 2015

Accepted for Publication: $31^{15}$ Dec 2015
In the course of enteric fever various organs can beinvolved leading to a wide range of presentation from uncomplicated typhoid fever to a complicated one involving multiple organs The hematological and biochemical changes due to multiorgan involvement in typhoid fever are not uncommon [1].

The hematological changes are common in typhoid fever and these include anaemia, leucopenia, eosinophilia, thrombocytopenia, elevated ESR and sub clinical disseminated intravascular coagulation.s [1,3].

Elevated prothrombin time (PT), Activated Partial Thromboplastin time (APPT) have also been reported [3]. This study was done to ascertain the degree of changes in some hematological parameters of typhoid patients as it is a multi systemic disease 


\section{Materials and Methods}

The present study was conducted on OPD and IPD patients in LLR Hospital Kanpur presenting to Emergency Pathology Lab, GSVM who were tested positive for typhoid by positive blood culture or positive widal test.

Inclusion Criteria: All patients diagnosed with typhoid fever were included in the study regardless of their age and sex. Patients with complaints of fever $>1$ week duration were investigated for typhoid. Diagnosis was confirmed by a positive culture of blood or widal test.

Exclusion Criteria: Patients who had started antibiotic treatment before presenting to our hospital were excluded. Patients suffering from other major systemic illness - history of liver disease, renal disease, hematological disorders, immunocompromised status (drugs/HIV), paratyphoid, malaria, recent history of
Research Article

drug intake which may alter the blood parameters, active alcoholics were excluded from the study. An informed consent was taken and a detailed clinical history was taken to rule out the confounding illnesses as listed above. Using $5 \mathrm{ml}$ disposable syringe, three $\mathrm{ml}$ of venous blood was drawn in from each patient for $\mathrm{CBC}$ and widal test along with sample for blood culture and biochemical investigations wherever indicated. Sample for $\mathrm{CBC}$ was collected in EDTA vial and hemogram was obtained using Medonic cell counter. A Leishman stained peripheral blood smear was prepared and studied in each case to study the general blood picture and presence of Plasmodium species. Widal test was performed by using serum separated from sample in plain vial. Salmonella typhi $\mathrm{O}$ and $\mathrm{H}$ agglutination titres $>1: 80$ and $>1: 160$ were considered to be significant and were included in the study as widal positive cases. Blood culture results were recorded where available. The data was tabulated for various parameters and analyzed statistically

\section{Results}

The study was conducted on 121 patients diagnosed with typhoid fever of which 43 patients were admitted patients who had severe systemic manifestations and complications like hepatomegaly (21 cases). Sex ratio was found to be almost equal with $50.5 \%$ male and $49.5 \%$ female patients. Maximum cases in male patients $(32.7 \%)$ were seen in $11-20 \mathrm{yr}$ age group while in females most common age group was $21-30 \mathrm{yrs}$ of age (38.3\%). The mean leucocyte count was $7.2 \mathrm{X} 10$ $9 / \mathrm{L}$, mean platelet count was $1.86 \mathrm{lakhs} / \mathrm{mm}^{3}$ and mean PCV was $29.6 \%$. In Differential count mean neutrophil percentage was $28 \%$ and mean lymphocyte percentage was $59 \%$ and mean eosinophil percentage was $2 \%$ Thrombocytopenia was observed in $39.7 \%$. leucopenia in $11.6 \%$ of cases and anemia was noted in $58.7 \%$ of total cases. This data reflects overall status of anemia which may be attributed to underlying malnutrition and which is further aggravated by coexisting infection.

Table 1: Age and sex wise distribution of patients

\begin{tabular}{|c|c|c|}
\hline Age Group & Male & Female \\
\hline $0-10$ & 11 & 06 \\
\hline $11-20$ & 20 & 20 \\
\hline $21-30$ & 15 & 23 \\
\hline $31-40$ & 10 & 10 \\
\hline $41-50$ & 03 & 03 \\
\hline $51-60$ & 00 & 00 \\
\hline$>60$ & 04 & 01 \\
\hline
\end{tabular}

Table 2: Variation in platelet count among patients under study

\begin{tabular}{|c|c|c|}
\hline Platelet count/cu mm & Male & Female \\
\hline$<50,000$ & 01 & 06 \\
\hline $0.5-.99$ lacs & 06 & 04 \\
\hline $1.0-1.49$ lacs & 17 & 14 \\
\hline$>1.5$ lacs & 37 & 36 \\
\hline
\end{tabular}


Research Article

Table 3: Variation in leucocyte count among patients under study

\begin{tabular}{|c|c|c|}
\hline TLC(mm3) & Male & Female \\
\hline$<4,000$ & 08 & 06 \\
\hline $4,000-11,000$ & 38 & 47 \\
\hline$>11,000$ & 15 & 07 \\
\hline
\end{tabular}

Table 4: Variation in haemoglobin level among patients under study

\begin{tabular}{|c|c|c|}
\hline Hemoglobin gms/dl & Male & Female \\
\hline$<10$ & 32 & 39 \\
\hline $10-12$ & 18 & 19 \\
\hline$>12$ & 11 & 02 \\
\hline
\end{tabular}

Mean Platelet Count - 1.86 lakhs $/ \mathrm{mm}^{3}$

Mean Leucocyte count $-7.2 \times 10^{9} / \mathrm{L}$

Mean Packed Cell Volume - 29.6\%

\section{Discussion}

Salmonella typhi remains a serious problem in developing countries. It continues to be a major cause of morbidity and mortality in tropical countries, especially among children [4]. The bacilli spread via contaminated food, drink or water. Typhoid fever usually arises due to lack of personal hygiene.

It is comman in places where there is poor sanitation, but especially where the water supply is liable to be contaminated by human excreta [2]. It is a systemic infection which can present in a multitude of ways. Characteristic presenting features include fever, relative bradycardia, diarrhea or constipation and abdominal pain [5].

Since the bacilli are found in blood, faeces and urine of both ill persons and asymptomatic carriers, diagnosis may be confirmed by laboratory tests that include isolation of S.typhi from blood, stool and urine specimens and by specific agglutination of S. typhi with the patients blood serum known as widal reaction [2]. In the progression of typhoid fever the second week is commonly described as the week of complications [5].

Chiu Cheng Hsun et al reported thrombocytopenia as most common complication in their study. Other complications were intestinal perforation, (3\%) rectal bleeding (3\%), ascitis or pleural effusion (4\%) and meningitis $(1 \%)$. [6].

Neurological complications such as meningism, delirium, coma and convulsions have been reported.
[5]. A pediatric population study by Chiu Cheng Hsun et al reported that incidence of complications tends to be higher among children 5years of age or older [6]. Chow et al investigated usefulness of widal test in diagnosing typhoid fever in endemic areas in children. They found widal test to be positive in $88 \%$ of typhoid fever cases on $1^{\text {st }}$ occasion when the test was done. [7]. Parry et al also reported that $83 \%$ of blood culture positive cases of typhoid fever had a positive widal test [8].

Few studies have been conducted on hematological profile of typhoid fever patients with no reported study in our region. Akgun et al reported leucopenia in $20 \%$ of patients. [9] Yaramis A et al [4] reported leucopenia in $18 \%$ \& thrombocytopenia in $10 \%$ of cases in their study on pediatric population.

They reported a left shift in $78 \%$ of cases. Leucocyte count is usually not less than $2500 / \mathrm{mm}^{3}$ and severe leucopenia (less than $2000 / \mathrm{mm}^{3}$ ) is very rare [7]. Leucocytosis is commonly seen in children in first 10 days of illness \& in cases of hemorrhage. P K Yap \& C $\mathrm{T}$ Chua [11] observed anemia in $13 \%$, leucopenia in 16 $\%$ \& thrombocytopenia in $32 \%$ of cases in their study.

Malik A S \& Malik R H [12] found thrombocytopenia in $26 \%$ of typhoid fever cases in Malasian children. Ifeanyi $\mathrm{O}$ E reported reduced PCV, reduced neutrophil count and relatively raised lymphocyte count in typhoid patients [3]. Thrombocytopenia is generally seen as a 
Research Article

complication of typhoid fever. However it can also be encountered as a presenting symptom [13].

The hematological changes are common in typhoid fever and these include anemia, leucopenia, eosinophilia, thrombocytopenia and sub clinical disseminated intravascular coagulation. Bone marrow suppression and hemophagocytosis are considered to be an important mechanism in producing hematological changes [1].

Toxic marrow suppression especially during initial septicemia phase of infection is believed to be a cause of thrombocytopenia [5,11]. The occurrence of anemia, leucopenia \& thrombocytopenia is attributed to invasion of hematopoietic organs by S. typhi causing depression of haematopoiesis [2].

The bone marrow of typhoid patients shows myeloid maturation arrest, decrease in number of erythroblasts and megakaryocytes with increased phagocytic activity of histiocytes [14]. Thrombocytopenia may contribute to prolonged bleeding lesions in the intestinal tract with consequent danger of hemorrhage and intestinal wall perforation [2]. Complete recovery is expected following successful treatment of the underlying infection [5].

From a practical point of view the association of fever with thrombocytopenia in typhoid fever is important in our country where various viral infections like dengue are common \& present with overlapping complaints [5]. Such patients should not be dismissed out of hand and the more sinister illness should be excluded. [11]. Stark et al have reported that single qualitative dengue serology testing carry false positive rate of up to $42.5 \%$. As such withdrawal of appropriate antibiotics in such a case would be detrimental [15]. So, a high index of suspicion is needed for not missing a diagnosis of typhoid fever with atypical features at presentation. We conclude that among association of leucopenia and thrombocytopenia with typhoid fever, thrombocytopenia has not been yet emphasized as a associated fever of typhoid fever. In tropical countries where typhoid is endemic, awareness of this relationship is necessary for prompting correct diagnosis and successful cure of patients.

\section{Acknowledgements: Nil}

Conflicts of interest: The authors declare that there are no conflicts of interest

Funding: Nil

\section{References}

1. Abro AH, Abdou AMS, Gangwani JL, Ustadi AM, Younis NJ, Hussaini HS. Haematological and biochemical changes in typhoid fever. Pakistan Journal of Medical Sciences.2009; 25(2): 166 - 171.

2. Okafor A I.Haematological alterations due to typoid fever in Enugu Urban- Nigeria. Malaysian Journal of Microbiology.2007;3(2): 19-22.

3. Ifeanyi OE. Changes in some haematological parameters in typhoid patients attending University Health Services Department of Michael Okpara University of Agriculture, Nigeria. Int. J.Crr. Microbiol. App. Sci 2014; 3(1):670 -674

4. Yaramis A, Yildirim I, Katar S, Ozbek MN, Yalcin I, Tas $\mathrm{M}$ et al. Clinical and Laboratory Presentation of Typhoid fever. International Pediatrics.2001;16(4): 227 $-231$.

5. B. M. K. Cheong. Typhoid Fever Presenting as Acute Cerebellar Ataxia and severe Thrombocytopenia. The Medical Journal of Malaysia. 2008; 63 (1): 77-78.

6. Chiu CH, Tsai JR, Ou JT, Lin TY. Typhoid fever in children: a fourteen-year experience. Acta Paediatr Taiwan. 2000 Jan-Feb;41(1):28-32.

7. Chow CB, Wang PS, Cheung MW, Yan WW, Leung NK. Diagnostic value of the Widal test in childhood typhoid fever. Pediatr Infect Dis J. 1987 Oct;6(10):9147.

8. Parry CM, Hoa NT, Diep TS, Wain J, Chinh NT, Vinh H, Hien TT, White NJ, Farrar JJ. Value of a single-tube widal test in diagnosis of typhoid fever in Vietnam. J Clin Microbiol. 1999 Sep;37(9):2882-6.

9. Akgun Y, Bac B, Boylu S, Aban N, Tacyildiz I. Typhoid enteric perforation. $\mathrm{Br} \quad \mathrm{J}$ Surg. 1995 Nov;82(11):1512-5.

10. Khalili Matinzadeh Z. . Leucopenia and Thrombocytopenia in typhoid fever and a case report. Journal of Sabzevar School of Medical Sciences.2001; 3(21)): $83-87$.

11. Yap PK, Chua CT. The haemogram in the diagnosis of acute typhoid fever--with special reference to thrombocytopenia. Singapore Med J. 1983 Jun;24(3):161-2. 
Research Article

12. Malik AS, Malik RH. Typhoid fever in Malaysian children. Med J Malaysia. 2001 Dec;56(4):478-90.

13. Serefhanoglu K, Kaya E, Sevinc A, Aydogdu I, Kuku I, Ersoy Y. Isolated thrombocytopenia: the presenting finding of typhoid fever. Clin Lab Haematol. 2003 Feb;25(1):63-5.
14. Khosla SN, Anand A, Singh U, Khosla A. Haematological profile in typhoid fever. Trop Doct. 1995 Oct;25(4):156-8.

15. Wichmann O, Stark K, Shu PY, Niedrig M, Frank C, Huang JH, Jelinek T. Clinical features and pitfalls in the laboratory diagnosis of dengue in travellers. BMC Infect Dis. 2006 Jul 21;6:120.

\section{How to cite this article?}

Shrivastava K, Vahikar S, Mishra V. Hematological profile in typhoid fever. Trop J Path Micro 2015;1(1):16-20.doi: 10.17511/jopm.2015.i01.04 